\title{
Electronic and Magnetic Structure of Transition-Metal Carbodiimides by Means of GGA $+U$ Theory
}

\author{
Hongping Xiang, ${ }^{\dagger}$ Richard Dronskowski, ${ }^{*},{ }^{\dagger}$ Bernhard Eck, ${ }^{\dagger}$ and Andrei L. Tchougréeff ${ }^{\dagger, \ddagger}$ \\ Institute of Inorganic Chemistry, RWTH Aachen University, Landoltweg 1, D-52056 Aachen, Germany, and \\ Poncelet Laboratory, Independent University of Moscow, Moscow Center for Continuous Mathematical \\ Education, Bolshoi Vlasevsky Per. 11, 119002 Moscow, Russia
}

Received: August 26, 2010

\begin{abstract}
The electronic structures and magnetic properties of $\mathrm{MNCN}(\mathrm{M}=\mathrm{Fe}, \mathrm{Co}$, and $\mathrm{Ni}$ ) have been investigated by density-functional theory including explicit electronic correlation through an ad hoc Coulomb potential $(\mathrm{GGA}+U)$. The results evidence $\mathrm{CoNCN}$ and $\mathrm{NiNCN}$ as type-II anti-ferromagnetic semiconductors (that is, intralayer ferromagnetic and interlayer anti-ferromagnetic), in accordance with experimental observations. Just like the prototype $\mathrm{MnNCN}$, the $\mathrm{MNCN}$ phases, with $\mathrm{M}=\mathrm{Ni}$ and $\mathrm{Co}$, thus resemble the corresponding MO monoxides with respect to their magnetic and transport properties. By contrast, FeNCN remains (semi)metallic even upon applying a strong Coulomb correlation potential. This, most probably, is in contradiction with its observed optical transparency and expected insulating behavior and points toward a serious density-functional theory problem.
\end{abstract}

\section{Introduction}

The transition-metal monoxides $\mathrm{MnO}, \mathrm{FeO}, \mathrm{CoO}$, and $\mathrm{NiO}$ hold an exclusive position in (theoretical) condensed-matter science because they represent the well-known archetypes for studying the fundamental nature of insulating states and magnetic interactions. Under standard conditions, their crystal structure is of the $[\mathrm{NaCl}]$ (rock-salt) type with space group $F m \overline{3} m$, but close to the Néel temperature $\left(T_{\mathrm{N}}\right)$, where the typeII anti-ferromagnetic order - that is, ferromagnetic within the (111) plane and anti-ferromagnetic between the (111) planesinstalls, a small rhombohedral distortion from the cubic symmetry is observed as well. ${ }^{1}$ Chemical intuition suggests that the bonding in these phases results from the interaction between originally fully occupied oxygen $2 p$ levels, empty metal $4 \mathrm{~s}$ levels, and partially occupied $3 \mathrm{~d}$ levels, but the insulating behavior can not be interpreted within the simple conceptual framework of one-particle band theory. ${ }^{2}$ Common text-book description focuses on the scenario of Mott insulators ${ }^{3-6}$ due to the on-site Coulomb correlations of the $3 \mathrm{~d}$ electrons. Nonetheless, there are unresolved controversies in terms of the disputable character of the top of the valence bands as seen from the perspective of both experiment and numerical modeling. ${ }^{7-16}$ We note that besides the electron correlations the rhombohedral distortion itself has also been proposed to assist in opening a gap at the Fermi level in the low-temperature phases undergoing anti-ferromagnetic ordering (see above). ${ }^{17}$ With the passage of time it became more or less a common point of view that the formation of the anti-ferromagnetic state according to the Mott-Hubbard scenario is responsible for the observed insulating behavior of transition-metal oxides. The rhombohedral distortion taking place in oxides below $T_{\mathrm{N}}$ has been said to significantly change the nature of the highest filled bands. ${ }^{18}$ Thus, it is probably fair to say that the microscopic

* To whom correspondence should be addressed. E-mail: drons@ hal9000.ac.rwth-aachen.de.

${ }^{\dagger}$ RWTH Aachen University.

* Independent University of Moscow. origin of the insulating behavior of the transition-metal monoxides and the exact nature of the states on top of the valence band still remains a fundamental, but to a significant extent unsettled, question.

The recently reported $3 \mathrm{~d}$ transition-metal carbodiimides MNCN may be viewed as the nitrogen-based analogs of the aforementioned monoxides. In the MNCN phases, the doubly charged "organic" carbodiimide anion $\mathrm{NCN}^{2-}$ takes the role of the oxygen dianion $\mathrm{O}^{2-}$, such that one might expect similarities with the corresponding monoxides both in terms of crystal structures and magnetic properties. ${ }^{19-22}$ With respect to the latter, all magnetic carbodiimides include transition-metal ions with high-spin electronic configurations, and the phases exhibit antiferromagnetic ordering for $\mathrm{M}=\mathrm{Mn}, \mathrm{Fe}, \mathrm{Co}$, and $\mathrm{Ni}$.

Both groups of compounds obviously manifest strong electronic correlations in their $3 \mathrm{~d}$ shells, and their theoretical inclusion is a prerequisite for a proper understanding. On the other hand, the amount of metal-nonmetal orbital mixing, or hybridization, that can be considered as a counterpoise for the correlations of basically intraatomic origin increases while going from oxides to carbodiimides because the electronegativity differences decrease upon moving from the $\mathrm{M}-\mathrm{O}$ to $\mathrm{M}-\mathrm{N}$ bonds, introducing more covalency.

With respect to structure we reiterate that the transition-metal monoxides in the type-II anti-ferromagnetic state below $T_{\mathrm{N}}$ undergo a rhombohedral distortion away from cubic symmetry, which induces the local symmetry reduction of the $\mathrm{MO}_{6}$ octahedra from $O_{h}$ to $D_{3 d}$, and this also changes the character of the states on top of the filled bands. ${ }^{18}$ In the transition-metal carbodiimides, however, the $\mathrm{MN}_{6}$ octahedra already correspond to $D_{3 d}$ symmetry even above $T_{\mathrm{N}}$ due to the spatial arrangement of the rod-like $\mathrm{NCN}^{2-}$ anions. Because of that, we consider the transition-metal carbodiimides as a helpful example allowing to single out the effect of the crystalline structure on the behavior of the energy gap and, also, on the composition of the states at the top of the filled band (provided it occurs).

In the present paper, we shall use the GGA $+U$ method for calculating the electronic ground states of the iso-structural 


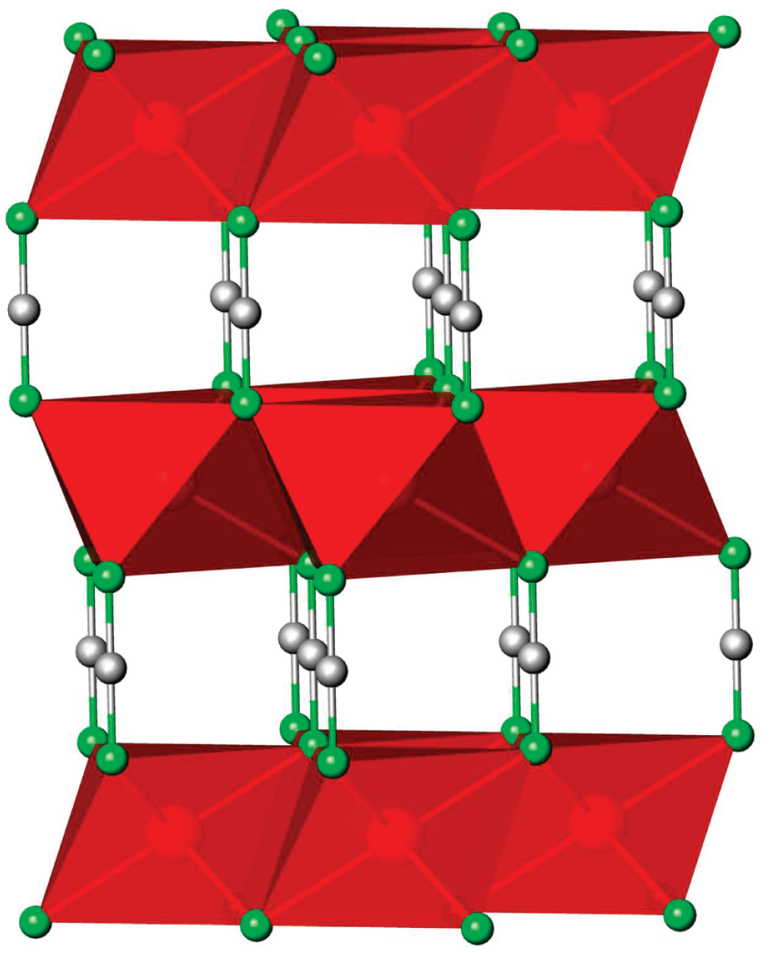

Figure 1. Crystal structure of $\mathrm{MNCN}(\mathrm{M}=\mathrm{Fe}, \mathrm{Co}$, and $\mathrm{Ni})$, with octahedral nitrogen (in green) coordination around $\mathrm{M}$ (in red) and linearly coordinated carbon atoms (in gray).

transition-metal carbodiimides $\mathrm{MNCN}(\mathrm{M}=\mathrm{Fe}, \mathrm{Co}$, and $\mathrm{Ni}){ }^{21,22}$ $\mathrm{X}$-ray powder data unambiguously manifest that these compounds adopt the [NiAs] structure type in the hexagonal system with space group $P 6_{3} / m m c$ (Figure 1 ). Here, the $\mathrm{M}$ atom is octahedrally coordinated by nitrogen atoms while the carbodiimide group experiences a trigonal-prismatic coordination by the metal atoms. Interestingly enough, this [NiAs] type of crystal structure is also found for both $\mathrm{FeO}$ and $\mathrm{CoO}$, but only at high pressures. ${ }^{23,24}$

As said before, all three phases are anti-ferromagnetic, just like the monoxides, and their Néel temperatures are $345 \mathrm{~K}$ (FeNCN), $255 \mathrm{~K}(\mathrm{CoNCN})$, and $360 \mathrm{~K}$ (NiNCN). Their transparent optical appearance (FeNCN: dark-red, ${ }^{22}$ CoNCN: orange-brown, ${ }^{21} \mathrm{NiNCN}$ : light-brown ${ }^{21}$ ) indicates that they are extremely likely to behave as electric insulators. Their conductivities and precise anti-ferromagnetic structures, however, have not been experimentally examined yet. In this contribution we will thus theoretically study (and eventually predict) the electronic and magnetic structures of $\mathrm{MNCN}(\mathrm{M}=\mathrm{Fe}, \mathrm{Co}$, and $\mathrm{Ni}$ ) with use of density-functional theory including explicit electron correlation. The type of ground state and of magnetic ordering (if any) will be studied, as well as the composition of the electronic states in the vicinity of the gap.

\section{Theoretical Calculations}

All calculations were based on density-functional theory (DFT) $)^{25,26}$ and performed by means of the plane-wave-pseudopotential Vienna ab initio Simulation Package (VASP). ${ }^{27-29}$ For the exchange-correlation functional, the generalized-gradient approximation as formulated by Perdew, Burke, and Ernzerhof $(\text { GGA-PBE })^{30}$ was employed. The projector-augmented wave (PAW) method as proposed by Blöchl ${ }^{31}$ and implemented by Kresse and Joubert ${ }^{32}$ was used, with a cutoff energy of $400 \mathrm{eV}$. A mesh of $9 \times 9 \times 3 k$-points was used to sample the complete Brillouin zone, and a finer one of $11 \times 11 \times 5 k$-points to calculate the densities of states. The Brillouin zone integrations were carried out with the tetrahedron method and Blöchl's correction to that. ${ }^{33}$ The PAW pseudopotentials considered in this study are $3 d^{6} 4 s^{2}$ for $\mathrm{Fe}, 3 d^{7} 4 s^{2}$ for $\mathrm{Co}, 3 d^{8} 4 s^{2}$ for $\mathrm{Ni}, 2 \mathrm{~s}^{2} 2 \mathrm{p}^{3}$ for $\mathrm{N}$, and $2 \mathrm{~s}^{2} 2 \mathrm{p}^{2}$ for $\mathrm{C}$ atoms, respectively. Electron-electron Coulomb interactions for the transition elements in combination with the self-interaction correction were considered in the rotationally invariant way $(\mathrm{GGA}+U)$ with a single effective Hubbard parameter $U_{\text {eff }}=U-J .^{34,35}$ The lattice parameters and atomic position were taken from experiment ${ }^{21,22}$ and further kept unchanged while the electronic self-consistency was reached.

In the $\mathrm{MNCN}(\mathrm{M}=\mathrm{Fe}, \mathrm{Co}$ and $\mathrm{Ni})$ phases considered here the $\mathrm{NCN}^{2-}$ units adopt ideal $D_{\infty h}$ symmetry of a free carbodiimide dianion, that is, a linear $\mathrm{N}-\mathrm{C}-\mathrm{N}$ group with two $\mathrm{C}=\mathrm{N}$ double-bonds, which arrive at 1.217(11) $\AA$ for FeNCN, 1.226(2) $\AA$ for CoNCN, and 1.233(16) $\AA$ for NiNCN. The carbodiimide units are coordinated by six $\mathrm{M}^{2+}$ cations in a trigonal-prismatic fashion. ${ }^{21,22}$ The $\mathrm{M}^{2+}$ ions are also bonded to six $\mathrm{NCN}^{2-}$ anions, but here one finds octahedra slightly flattened along the 3-fold axis that is parallel to the crystallographic $c$ axis. The interatomic separations are: $\mathrm{Fe}-\mathrm{N}=2.201(6) \AA, \mathrm{Co}-\mathrm{N}=2.168(1) \AA$, and $\mathrm{Ni}-\mathrm{N}=2.119(8) \AA{ }^{21,22}$ mirroring the course of the ionic radii for 6-fold coordination. ${ }^{36}$ Figure 1 displays that the $\mathrm{MN}_{6}$ octahedra engage in edge-sharing within the $a b$ plane and are further connected by $\mathrm{N}=\mathrm{C}=\mathrm{N}^{2-}$ units along the $c$ axis, thereby forming a formally layered structure. A closer look at the carbodiimides reveals that the octahedral side-lengths (distance between two neighboring $\mathrm{N}$ atoms) and $\mathrm{N}-\mathrm{M}-\mathrm{N}$ angles are $2.95 / 3.27 \AA(\Delta=0.32 \AA)$ and $84.1 / 95.9^{\circ}\left(\Delta=11.8^{\circ}\right)$ for $\mathrm{FeN}_{6}$, $2.91 / 3.21 \AA(\Delta=0.30 \AA)$ and $84.4 / 95.6^{\circ}\left(\Delta=11.3^{\circ}\right)$ for $\mathrm{CoN}_{6}$, and $2.83 / 3.15 \AA(\Delta=0.32 \AA)$ and $83.9 / 96.1^{\circ}\left(\Delta=12.3^{\circ}\right)$ for $\mathrm{NiN}_{6}$, indicating that the distortions of the $\mathrm{FeN}_{6}, \mathrm{CoN}_{6}$, and $\mathrm{NiN}_{6}$ octahedra away from ideal $O_{h}$ symmetry are similar in magnitude.

The GGA $+U$ method is an established DFT-style method to numerically treat strongly correlated $3 \mathrm{~d}$ electrons. It eventually allows for an artificial localization of the delocalized electronic states that manifests in the formation of spin densities interpreted as local momenta. Some semiempirism is involved because it is fairly difficult to independently estimate a reasonable $U$ parameter. Since in the present MNCN systems the energy gap is not available, the $U$ parameter was taken from previous studies on the transition-metal oxides $(U=7 \mathrm{eV}$ for $\mathrm{Ni}$ and $\mathrm{Co}$; $U=$ $6 \mathrm{eV}$ for $\mathrm{Fe}){ }^{37}$

\section{Results and Discussion}

Magnetic Structures and Effective Exchange Parameters. On structural considerations one expects two effective magnetic interactions for MNCN, $J_{1}$ (six neighbors) and $J_{2}$ (two neighbors) between the "nearest-neighbor" metal ions as corresponding to the $\mathrm{M}-\mathrm{N}-\mathrm{M}$ and $\mathrm{M}-\mathrm{N}=\mathrm{C}=\mathrm{N}-\mathrm{M}$ superexchange paths, respectively (see Figure 2a). In order to estimate the magnitudes of the effective exchange parameters, we considered a ferromagnetic (FM) and three anti-ferromagnetic structures (AFM1, AFM2, and AFM3), also shown in Figure 2, needed to identify the most probable magnetic structure. In the AFM1 scenario, $J_{1}$ corresponds to a ferromagnetic coupling, whereas $J_{2}$ is antiferromagnetic as in the type-II anti-ferromagnetic phases of MO $(\mathrm{M}=\mathrm{Fe}, \mathrm{Co}$ and $\mathrm{Ni}$ ) in which the couplings between the nearest-neighbor metal ions are ferromagnetic and anti-ferromagnetic for the $\mathrm{M}-\mathrm{O}-\mathrm{M}$ angles of 90 and $180^{\circ}$, respectively. ${ }^{1}$ This can be considered analogous to the coupling along the $\mathrm{M}-\mathrm{N}-\mathrm{M}$ and $\mathrm{M}-\mathrm{N}=\mathrm{C}=\mathrm{N}-\mathrm{M}$ superexchange paths, respectively. In addition, a $2 \times 1 \times 1$ supercell was used to build the 


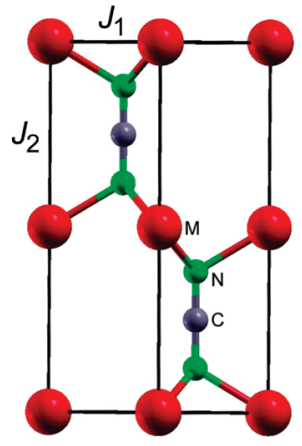

a) FM

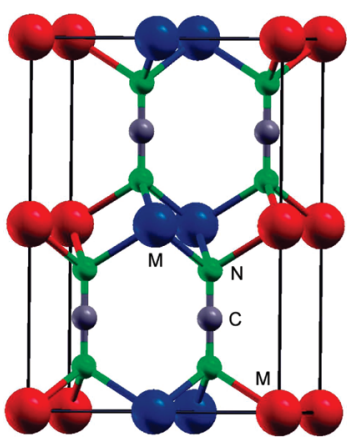

c) AFM2

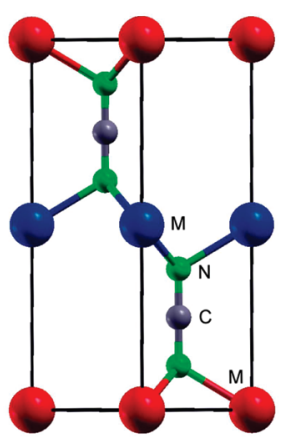

b) AFM1

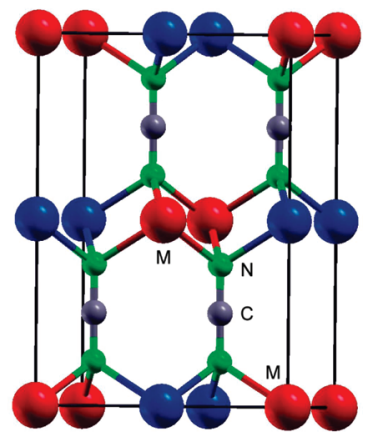

d) AFM3
Figure 2. Theoretically investigated ordered spin arrangements of MNCN (M = Fe, Co, Ni) designated as (a) FM, (b) AFM1, (c) AFM2, and (d) AFM3. Red and blue spheres indicate different orientations of the spin moments residing on the transition-metal $\mathrm{M}$ atoms.

AFM2 model-ferromagnetic within the $a c$ plane and antiferromagnetic between the ac planes - and the AFM3 modelferromagnetic within the (202) plane and anti-ferromagnetic between the (202) planes.

The total energies as calculated for the adopted $U$ values evidence that an II-type anti-ferromagnetic structure (intralayer ferromagnetic within $a b$ plane, interlayer anti-ferromagnetic between the $a b$ planes; see AFM1 in Figure 2b) is most stable, in agreement with the anti-ferromagnetism as derived from the susceptibility measurements. ${ }^{21,22}$ The theoretical anti-ferromagnetic structures of FeNCN, CoNCN, and NiNCN thus resemble those of $\mathrm{FeO}, \mathrm{CoO}$, and $\mathrm{NiO} .{ }^{1}$ The comparison of the GGA $+U$ energies of various magnetic phases allows estimating the magnitudes of the effective exchange parameters, which are summarized in Table 1. Remarkably enough, the simple GGA calculation $(U=0)$ yield the same order of different magnetic states on the energy scale. The comparison of the calculated spin densities with the results of magnetic measurements, however, requires a more complex approach. Although the spin densities can be directly compared with the saturation magnetic momenta $\mu_{\text {sat }}$ (to be measured by neutron diffraction) in the lowtemperature magnetically ordered phase, such data are not yet available for the compounds under study. Only the effective momenta $\mu_{\mathrm{eff}}^{*}$ measured right above the Néel temperature are known to us. ${ }^{21,22}$ The relation between them may be expressed by the following formula (for details see Appendix A):

$$
\mu_{\mathrm{sat}}=\sqrt{\frac{2}{\left(1-\frac{J_{2}}{3 J_{1}}\right)\left(1+\frac{1}{S}\right)}} \mu_{\mathrm{eff}}^{*}
$$

With these precautions, let us cover the MNCN phases in detail. For pedagogical reasons we proceed from right to left, that is, reverse to the order of the Periodic Table. The effective paramagnetic moment $\mu_{\mathrm{eff}}^{*}$ of NiNCN at the Néel point is 1.8 $\mu_{\mathrm{B}}{ }^{21}$ The saturation moment derived from it by the above formula then gives $1.71 \mu_{\mathrm{B}}$, which remarkably coincides with the value of $1.67 \mu_{\mathrm{B}}$ obtained numerically at $U=7 \mathrm{eV} .{ }^{37}$ The effective paramagnetic moment $\mu_{\mathrm{eff}}^{*}$ of CoNCN at $255 \mathrm{~K}\left(T_{\mathrm{N}}\right)$ is about $3.1 \mu_{\mathrm{B}} \cdot{ }^{21}$ Recalculated into a saturation momentum this yields $3.29 \mu_{\mathrm{B}}$, in fair agreement with the GGA $+U$ calculated value of $2.75 \mu_{\mathrm{B}}$. Magnetic susceptibility measurements show that, above the Néel temperature of $345 \mathrm{~K}$, the effective paramagnetic moment $\mu_{\text {eff }}^{*}$ of FeNCN is about $3.9 \mu_{\mathrm{B}},{ }^{22}$ a bit larger than our calculated saturation moment of $3.67 \mu_{\mathrm{B}}$. The procedure used above to recalculate the saturation magnetic momentum from the susceptibility data at the Néel temperature yields $2.41 \mu_{\mathrm{B}}$, but it most probably does not apply since the interlayer anti-ferromagnetic exchange parameter turns out to be unrealistically strong as compared to the intralayer ferromagnetic one as provided by the GGA $+U$ procedure.

Electronic Structure, Gap, and Optical Properties. The results of the electronic-structure calculations as performed by use of GGA and GGA $+U$ are summarized in Figure 3. For the case of NiNCN, the GGA itself $(U=0)$ produces a band gap of $0.8 \mathrm{eV}$, as it also happens for the corresponding oxide ${ }^{37}(\Delta$ $=0.2 \mathrm{eV}$ ). Analogously to the oxide case this gap is too small to explain the transparency of the material for visible light. As a nonzero $U$ is applied, the band gap reaches a value of $2.7 \mathrm{eV}$, much smaller than the experimental gap (ca. $4.0 \mathrm{eV}$ ) of $\mathrm{NiO},{ }^{38}$ probably due to the larger covalent character. The projected DOS (Figure 3, lower row, right panel) demonstrate that the GGA $+U$ yields mainly N 2 p character for the higher filled bands, together with some minor mixing with the Ni d bands. By contrast, the lowest empty band is predominantly of $\mathrm{Ni} \mathrm{d}$ character, thus describing NiNCN as a charge-transfer insulator similar to NiO. ${ }^{13,14}$ Nevertheless, the description of the spectroscopic properties within GGA $+U$ must be assessed as rather poor: according to the theoretical model the absorption spectrum must have its edge almost at the ultraviolet border (460 nm vs 380 $\mathrm{nm}$ ) of the visible range.

Upon moving toward CoNCN, the simple GGA calculation yields a metallic ground state. When $U$ is set equal to $7 \mathrm{eV}$ as for $\mathrm{CoO}$ (an estimate from the constrained density-functional method), ${ }^{37}$ the band gap becomes $3.0 \mathrm{eV}$, which is somewhat larger (!) than the one of $\mathrm{CoO}(2.5 \mathrm{eV}){ }^{12}$ The DOS (Figure 3, lower row, middle panel) resembles the previous NiNCN case. The upper filled band is mostly made up from $\mathrm{N} 2 \mathrm{p}$ orbitals with insignificantly small hybridization with the Co $3 \mathrm{~d}$ bands; the lower empty bands, however, carry predominantly Co $3 \mathrm{~d}$ character, just like for NiNCN. Thus, the classification of CoNCN as a charge-transfer insulator also seems to be consistent. The band gap, however, is significantly overestimated, too, and it falls into contradiction even with the simplest optical properties: following GGA $+U$, the band edge of $\mathrm{CoNCN}$ must be even closer to the ultraviolet border $(415 \mathrm{~nm})$ of the visible range than for NiNCN.

The case of FeNCN, however, turns out to be a disaster scenario for the otherwise successful GGA $+U$ approach. First, the GGA $(U=0)$ results in a metallic ground state, just like in CoNCN. Applying $U$ based on constrained density-functional theory as previously for the related oxide phase $\mathrm{FeO}^{37}$ (in this case $U=6 \mathrm{eV}$ ) does not result in a gap opening since some noticeable density of states persists at the Fermi level. This is in clear contradiction with the optical properties since the material itself is transparent, apparently insulating, and in general not that much different from the $\mathrm{Co}, \mathrm{Ni}$, and $\mathrm{Mn}$ carbodiimides. 
TABLE 1: Total Energies of the FM, AFM1, AFM2, and AFM3 States Per Unit Cell Relative to That of the AFM1 State and the Effective Exchange Coupling Constants (all in meV) As Coming from the GGA and GGA $+U$ Calculations

\begin{tabular}{|c|c|c|c|c|c|c|c|}
\hline & & \multicolumn{2}{|c|}{ FeNCN } & \multicolumn{2}{|c|}{ CoNCN } & \multicolumn{2}{|c|}{$\mathrm{NiNCN}$} \\
\hline & & $U=0$ & $U=6 \mathrm{eV}$ & $U=0$ & $U=7 \mathrm{eV}$ & $U=0$ & $U=7 \mathrm{eV}$ \\
\hline \multirow[t]{3}{*}{ total energy } & FM & 308.7 & 1893.4 & 282.1 & 62.9 & 410.8 & 140.5 \\
\hline & AFM2 & 1097.7 & 966.2 & 1444.8 & 1277.5 & 1847.3 & 5619.2 \\
\hline & AFM3 & 1256.7 & 1284.7 & 1284.7 & 1189.9 & 1477.9 & 1291.6 \\
\hline coupling constant & $J_{1}$ & 68.6 & 63.2 & 80.3 & 74.4 & 92.4 & 80.7 \\
\hline
\end{tabular}

The above results may clarify the hypothetical role of the rhombohedral distortion in the gap formation as discussed for a long time. ${ }^{17}$ In the Ni case a small gap opens either in the ideal cubic symmetry (oxide ${ }^{37}$ ) or in the rhombohedrally distorted one due to chemical reasons (carbodiimide), even without any $U$. In both cases, adding $U$ increases the gap but not does not correctly describe the observed optical properties. In the Co case as considered by the GGA, no gap opens at the Fermi level, neither in the octahedral (oxide) nor in rhombohedral case (carbodiimide). Applying $U$ of the same strength as for Ni opens the gap, but the well is too large to describe the observed optical behavior. Finally, in the case of FeNCN neither the lower symmetry alone nor applying the Coulomb correlated potential completely removes the finite density of states from the Fermi level.

Summarizing the above findings we can conclude that the symmetry lowering caused by the rhombohedral distortion is
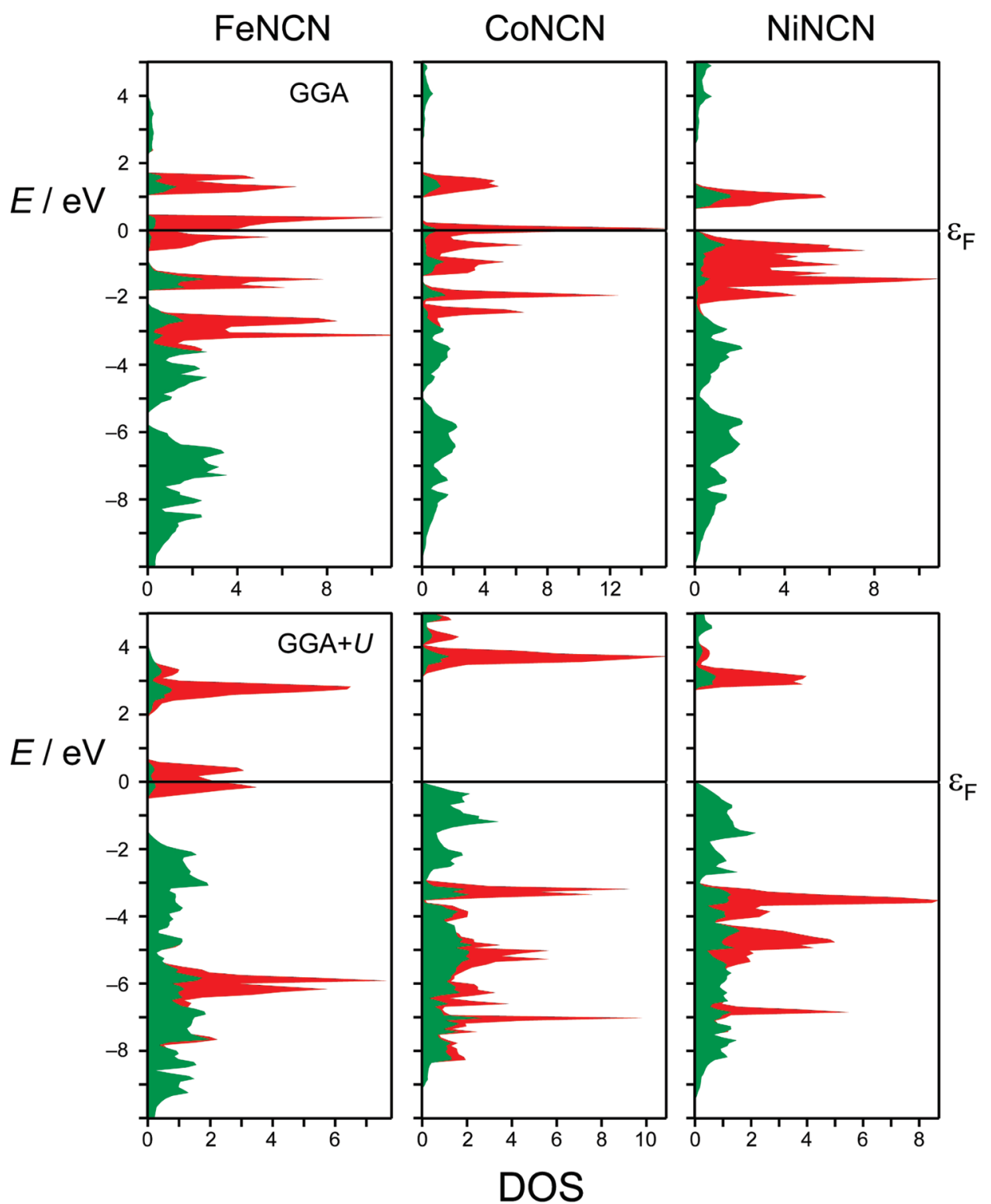

Figure 3. The densities-of-states of MNCN projected to transition-metal $3 \mathrm{~d}$ (red) and nitrogen $2 \mathrm{p}$ (green) orbitals on the basis of GGA (upper row) and GGA $+U$ (lower row) calculations. Carbon is not shown due to its insignificant contribution in the given energy window. The frames refer to FeNCN (left), CoNCN (middle), and NiNCN, (right). 
not the source for the gap opening in the transition-metal oxides ${ }^{17}$ as suggested, in particular because there is no gap opening in the case of the much stronger rhombohedral distortions dictated by the carbodiimide structures.

To better understand what is going on with the d orbitals in carbodiimides upon applying the Coulomb correlation potential we consider the transformations of the corresponding projected densities of states in more detail. Before doing so, we recall some symmetry issues as related to the analysis of the rhombohedral distortion.

For $\mathrm{M}^{2+}$ ions in an ideal $O_{h}$ symmetry of the $\mathrm{MO}_{6}$ octahedra in oxides, the d states are split by the ligand field into the $t_{2 g}$ and $e_{g}$ subsets with $E\left(e_{g}\right)-E\left(t_{2 g}\right)=\Delta>0$. This splitting applies equally to electrons of either spin projection, although the amount of splitting may be somewhat different. The rhombohedral distortion results in a symmetry lowering from $O_{h}$ to $D_{3 d}$, namely a contraction or elongation of the octahedron along one of its 3-fold axes. Such effects are conveniently described in so-called "trigonal" basis, where the $z$ axis coincides with the 3 -fold axis of the octahedron. Here, the states of the lower $t_{2 g}$ subset have the form ${ }^{39}$

$$
\left|z^{2}\right\rangle, \sqrt{\frac{2}{3}}\left|x^{2}-y^{2}\right\rangle-\sqrt{\frac{1}{3}}|x y\rangle, \sqrt{\frac{2}{3}}|x y\rangle+\sqrt{\frac{1}{3}}|y z\rangle
$$

and those of the upper $e_{g}$ subset have the form

$$
\sqrt{\frac{2}{3}}|x z\rangle+\sqrt{\frac{1}{3}}\left|x^{2}-y^{2}\right\rangle,-\sqrt{\frac{2}{3}}|y z\rangle+\sqrt{\frac{1}{3}}|x y\rangle
$$

where the d electron states involved are the states of the trigonal basis. The crystal structure of carbodiimides is such that the crystallographic $c$ axis is precisely the one of the trigonal distortion of the $\mathrm{MN}_{6}$ octahedra. Within the $D_{3 d}$ symmetry the octahedral $t_{2 g}$ subset further splits into a nondegenerate $a_{1 g}$ state, which is the $\left|z^{2}\right\rangle$ state and a doubly degenerate $1 e_{g}$ state; the octahedral $e_{g}$ state turns out to have the $e_{g}$ symmetry as well now with respect to the $D_{3 d}$ point group, and thus we denote it as the $2 e_{g}$ state, also doubly degenerate. Since the $1 e_{g}$ and $2 e_{g}$ states are of identical symmetry, they can mix with each other. If the distortion is small, then the splitting of the octahedral $t_{2 g}$ subset and the mixing of $1 e_{g}$ and $2 e_{g}$ states are small as well. In this case the splitting between the $1 e_{g}$ (and as well the $a_{1 g}$ state) and $2 e_{g}$ states approximately equals that between the $t_{2 g}$ and $e_{g}$ states in the undistorted octahedron.

With these prerequisites the reconstruction of the predominantly $\mathrm{d}$ bands upon applying the Coulomb correlation potential $U$ is easily analyzed. The relevant projected densities of states given in Figure 4 are performed on symmetry grounds: we project densities in both spin channels on the orbitals of the $a_{1 g}$ symmetry (the $\mathrm{d}_{z^{2}}$ states with respect to the crystal coordinate frame) and project the sum of the densities of states in both the $1 e_{g}$ and $2 e_{g}$ states. One expects that the $a_{1 g}$ density comes out as a single peak whereas the $e_{g}$ density shows up as two peaks corresponding to the $1 e_{g}$ and $2 e_{g}$ states, respectively, separated by the crystal field energy $\Delta$. This simple picture is, however, only partially reproduced. As can be seen (Figure 4, upper row, right panel) for NiNCN in the GGA, the $a_{1 g}$ state is fully occupied in both spin channels (see the numerical populations obtained by integration up to the Fermi energy in Table 2). Nonetheless, the $a_{1 g}$ densities manifest not as single peaks but develop some fine structure, most probably due to mixing with different bands stemming from surrounding $\mathrm{N}$ atoms. The $e_{g}$ density in the minority-spin channel fairly shows two peaks separated by ca. $1.5 \mathrm{eV}$ of crystal field splitting. The spinmajority states are thus almost completely occupied; the value from Table 2 must be compared with the theoretical value of five, but some deviation must be allowed because of interaction with the neighboring $\mathrm{N}$ atoms. The peak of the empty $2 e_{g}$ states in the spin-minority channel can be nicely seen at ca. $1 \mathrm{eV}$ above the Fermi level. The average shift of the spin-minority states relative to the respective spin-majority states originating from the intra-atomic exchange ${ }^{37}$ (Stoner parameter)-which is the only source of the spin asymmetry in the GGA setting-can be estimated to ca. $2.0 \mathrm{eV}$ up to $2.5 \mathrm{eV}$.

A similar picture is observed for the other two carbodiimides. Both for CoNCN and FeNCN the unoccupied $2 e_{g}$ states in the spin-minority channel are nicely separated from the $1 e_{g}$ states by $1.4 \mathrm{eV}(\mathrm{Co})$ and $1.1 \mathrm{eV}(\mathrm{Fe})$, respectively, as a result of the crystal field splitting. Analogously to the case of NiNCN the spin-majority states are almost completely occupied by electrons. The situation in the minority-spin channel is less clear. First of all, the minority spin states seem to be somewhat overpopulated by electrons (see Table 2) against the expected populations conforming to a theoretical $\mathrm{d}^{7}\left(\mathrm{Co}^{2+}\right)$ and $\mathrm{d}^{6}\left(\mathrm{Fe}^{2+}\right)$ configuration. Moreover, the $a_{1 g}$ and $1 e_{g}$ states both represented by degenerated "twin-peak" features due to interactions with the nitrogen bands fall on the Fermi level so that the lower ones of each of "twins" are fully occupied, whereas the upper ones are cut approximately in halves by $\varepsilon_{\mathrm{F}}$. The trigonal distortion is apparently the smallest energy scale here; it is smaller than the splitting due to the nonequivalence of the $\mathrm{N}$ bands and, therefore, invisible in the graphs.

Applying the Coulomb correlation potential changes the picture significantly, since with the predefined values ${ }^{37}$ of $U$ the Coulomb correlation potential is the strongest player here. The overall picture of the electronic states radically changes, but some of its symmetry-imposed features remain stable and can be traced back to the GGA. For example, in the DOS of NiNCN (Figure 4, lower row, right panel) we cannot see the splitting of either $a_{1 g}$ or $1 e_{g}$ states due to the interactions with the $\mathrm{N}$ bands because of quite different energy scales. The true crystal field splitting is, however, observed in the majority-spin channel as the energy difference (ca. $2 \mathrm{eV}$ ) between the positions of two green peaks representing the $1 e_{g}$ and $2 e_{g}$ states. The relative position of the yellow peak in the majority-spin channel (the $a_{1 g}$ density) with respect to its closest green peak $\left(1 e_{g}\right.$ density) manifests the meager amount of the rhombohedral splitting. The same is found for the minority-spin channel from the relative positions of the almost superimposed red and blue peaks ( $1 e_{g}$ and $a_{1 g}$, respectively). Since the overall effect of the Coulomb correlation potential is the splitting of the empty and occupied states on the same atom, the most pronounced effect occurs in the minority spin channel. The energy separation between the occupied minority-spin $a_{1 g}$ and $1 e_{g}$ states and the empty minority-spin $2 e_{g}$ states amounts to almost the value of $U$ itself $(7 \mathrm{eV})$. The significant distortion of the band positions around the Fermi level by $U$ suppresses all kinds of hybridization/delocalization effects and brings the populations close to their theoretical values (see again Table 2). The overall picture thus fairly corresponds to that of a d $\mathrm{d}^{8}$ shell typical for the $\mathrm{Ni}^{2+}$ ions $\left(\mathrm{Ni}^{2+}: \mathrm{d}^{5 \uparrow} \mathrm{d}^{3} \downarrow\right)$ with two holes in the $2 e_{g}$ states either in the notation of the $D_{3 d}$ or $O_{h}$ group.

Essentially the same features can also found in the DOS of $\mathrm{CoNCN}$ as derived within the GGA $+U$ framework. The crystal field splitting is taken as an energy difference between the $1 e_{g}$ and $2 e_{g}$ peak positions in the majority-spin channel (green 


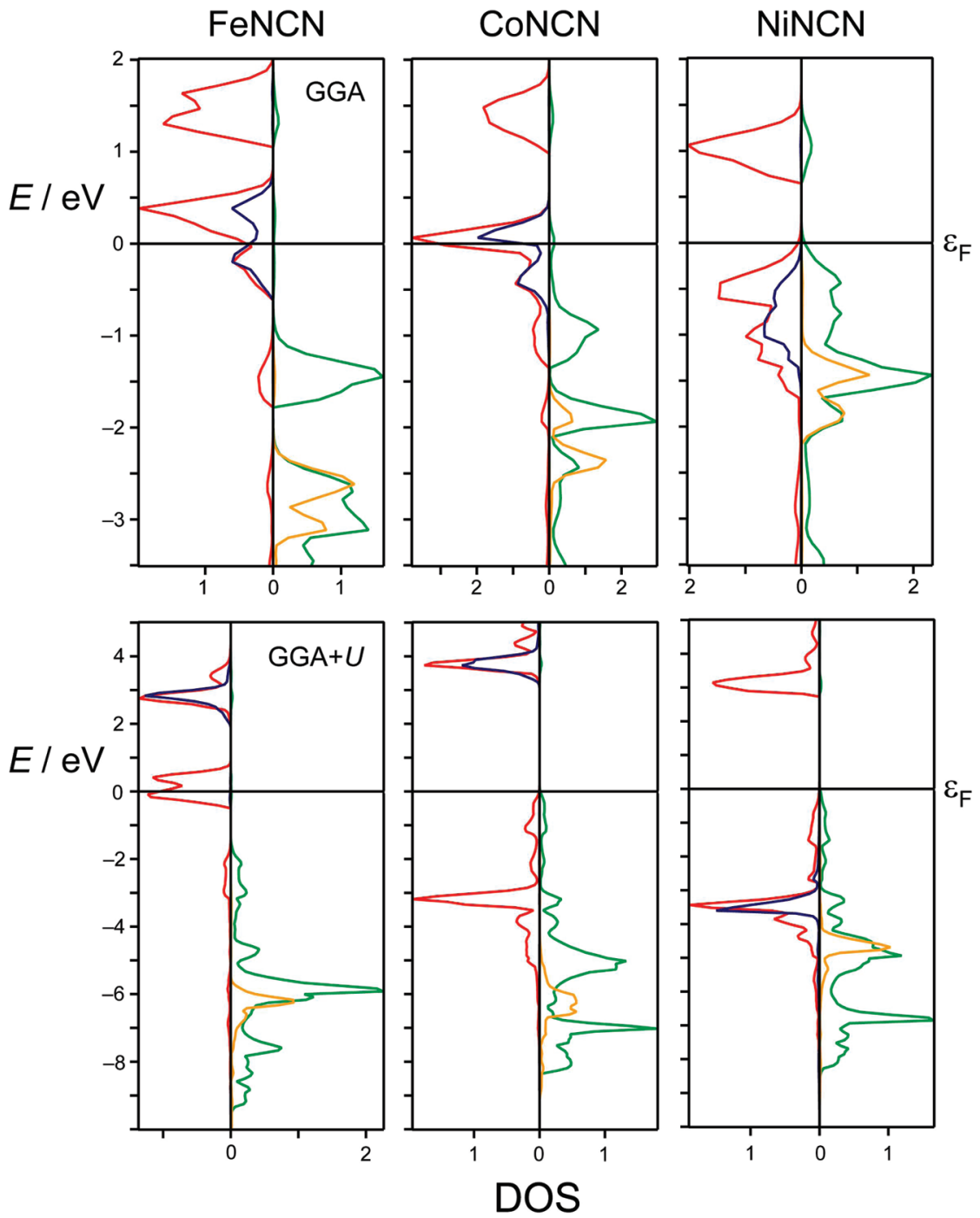

Figure 4. The orbital-projected densities-of-states in the spin majority (right half-panels) and spin-minority (left half-panels) of the metal $3 \mathrm{~d}$ orbitals on the basis of GGA (upper row) and GGA $+U$ (lower row) calculations for FeNCN (left), CoNCN (middle), and NiNCN (right). The colors (online) indicate $a_{1 g}$ projection (blue/yellow) and $e_{g}$ projection (red/green). See also text.

TABLE 2: Populations of the d Orbitals in the Majority- and Minority-Spin Channels for the $a_{1 \mathrm{~g}}$ and $e_{g}$ States As Calculated by Integration (up to $E_{\mathrm{F}}$ ) of the Projected Densities of States Coming from the GGA and GGA+U Calculations

\begin{tabular}{|c|c|c|c|c|c|c|c|c|c|c|c|c|c|}
\hline \multirow{3}{*}{\multicolumn{2}{|c|}{ Spin projection }} & \multicolumn{4}{|c|}{ FeNCN } & \multicolumn{4}{|c|}{$\mathrm{CoNCN}$} & \multicolumn{4}{|c|}{ CoNCN } \\
\hline & & \multicolumn{2}{|c|}{$U=0$} & \multicolumn{2}{|c|}{$U=6 \mathrm{eV}$} & \multicolumn{2}{|c|}{$U=0$} & \multicolumn{2}{|c|}{$U=7 \mathrm{eV}$} & \multicolumn{2}{|c|}{$U=0$} & \multicolumn{2}{|c|}{$U=7 \mathrm{eV}$} \\
\hline & & maj & $\min$ & maj & $\min$ & maj & $\min$ & maj & $\min$ & maj & $\min$ & maj & $\min$ \\
\hline \multirow[t]{3}{*}{ population } & $a_{1 g}$ & 0.996 & 0.385 & 0.699 & 0.012 & 0.956 & 0.713 & 0.972 & 0.012 & 0.943 & 0.765 & 0.863 & 0.956 \\
\hline & $e_{g}$ & 3.776 & 0.892 & 3.011 & 0.795 & 3.596 & 2.218 & 4.057 & 2.133 & 3.336 & 2.296 & 3.868 & 2.089 \\
\hline & $d$ & 4.772 & 1.277 & 3.710 & 0.807 & 4.552 & 2.931 & 5.029 & 2.145 & 4.279 & 3.06 & 4.731 & 3.045 \\
\hline
\end{tabular}

peaks), namely about $2 \mathrm{eV}$. The relative positions of the $a_{1 g}$ and $1 e_{g}$ peaks evidence, once again, the meager effect of the rhombohedral distortion. The total occupancy of the majorityspin states is close to ideal (see Table 2). As for the projected densities of states in the minority-spin channel, the qualitative consequences of this reconstruction are pretty much different. Without a finite $U$ (Figure 4, upper row, middle panel) the $a_{1 g}$ and $1 e_{g}$ states somewhat split due to the hybridization with the $\mathrm{N}$-bands and are partially occupied. Applying $U$ throws up the spin-minority $a_{1 g}$ state, which becomes almost degenerate with the empty $2 e_{g}$ states, whereas the spin-minority $1 e_{g}$ states become completely occupied. So far the picture fairly corresponds to that of the $\mathrm{d}^{7}$ shell typical for the $\mathrm{Co}^{2+}$ ions $\left(\mathrm{Co}^{2+}\right.$ : $\left.d^{5 \uparrow} d^{2} \downarrow\right)$ with three holes in three highest energy states.
Finally, we analyze the projected densities of states in two spin channels of the unsuccessful case of FeNCN. The amount of reconstruction upon applying $U$ in the majority spin channel and its pattern are similar to the NiNCN and CoNCN cases. A crystal field splitting shows upwards of ca. $2 \mathrm{eV}$, and the pure rhombohedral splitting is negligibly small. In the minority spin channel the $a_{1 g}$ state is thrown up as before, and it becomes empty and degenerate with the $2 e_{g}$ states. Most intriguing is the behavior of the minority spin $1 e_{g}$ states. A thorough inspection of the corresponding peak in the projected density of states (Figure 4, lower row, left panel) yields a small splitting so that its lower component is completely filled whereas the upper one is empty. Nevertheless, these two subbands do not go far away from the Fermi level, although one may also 
conjecture that the density depletion between the two small peaks just above/below the Fermi level indicates the formation of a small gap. Whether it exists or not, the aforementioned finding is in a strong contradiction with the most probable insulating behavior of FeNCN. The occupancy of a $1 e_{g}$ state rather of the $a_{1 g}$ is in a line with the level ordering in CoNCN: the $1 e_{g}$ state has a lower energy than the $a_{1 g}$ state.

\section{Conclusion}

The electronic and magnetic structures of the transition-metal carbodiimides $\mathrm{MNCN}(\mathrm{M}=\mathrm{Fe}, \mathrm{Co}$ and $\mathrm{Ni}$ ) have been calculated by use of GGA and GGA $+U$ with $U$ values taken to be $6 \mathrm{eV}$ for $\mathrm{Fe}$ and $7 \mathrm{eV}$ for $\mathrm{Co}$ and $\mathrm{Ni}^{37}{ }^{37}$ The results suggest the $\mathrm{Ni}$ and Co compounds to be type-II anti-ferromagnetic insulators, similar to the corresponding transition-metal monoxides. This magnetic behavior is also corroborated from the macroscopic magnetic measurements. ${ }^{21}$ Similar measurements show FeNCN be an anti-ferromagnet as well, but our calculations only partially reproduce this result: they show reasonable value of the saturation magnetic moment but no significant energy gap. The composition of the states bordering the gap in the $\mathrm{Co}$ and $\mathrm{Ni}$ carbodiimides indicates predominantly chargetransfer type of the low-energy excitations in these compounds, similar to what has been observed for the corresponding monoxides. The local electronic structures of the $\mathrm{M}^{2+}$ ions indicate that the majority-spin $3 \mathrm{~d}$ states are fully filled and separated from the minority spin states by the exchange splitting. Apparently, the rhombohedral distortion introduced by the chemical structure of the $\mathrm{NCN}^{2-}$ dianions and leading to the $D_{3 d}$ symmetry of the $\mathrm{MN}_{6}$ octahedra has no significant effect on the electronic structure of the MNCN phases. For the minority-spin levels, the electronic configurations of $a_{1 g}{ }^{1} e_{g}{ }^{0}$ for $\mathrm{Fe}^{2+}, a_{1 g}{ }^{0} e_{g}{ }^{2}$ for $\mathrm{Co}^{2+}$, and $a_{1 g}{ }^{1} e_{g}{ }^{2}$ for Ni would tentatively lead to the formation for a band gap between $a_{1 g}$ and $e_{g}$ levels. The required configurations are indeed observed in our calculations for the Ni and Co carbodiimides, but not for the Fe compounds, where one of two degenerate $e_{g}$ levels is occupied. This must be considered a serious failure of the GGA $+U$ approach, although is easily understandable by itself: the $1 e_{g}$ states have a lower energy than the $a_{1 g}$ ones so that the latter are more easily filled by the holes upon going from NiNCN to FeNCN. Remarkably enough this unphysical result is in agreement with the calculations previously performed on $\mathrm{FeO}:{ }^{18}$ if the Coulomb correlation potential and the rhombohedral distortion are applied together to $\mathrm{FeO}$, the corresponding DFT-based calculation predicts one of the components of the $1 e_{g}$ state to be occupied in the minority-spin channel. Such an electronic ground state must be considered as a prerequisite for further deformations due to Jahn-Teller distortion which, however, does not take place, neither in $\mathrm{FeO}$ nor in $\mathrm{FeNCN}$ as evident from the X-ray crystal structures. This allows us to conclude that the FeNCN case is one more significant problem for the DFT-based $\mathrm{GGA}+U$ treatment.

Acknowledgment. It is a pleasure to thank Prof. Dr. Heiko Lueken, Dr. Xiaohui Liu, and Dr. S. N. Zagoulyaev (St. Petersburg) for helpful discussions and Deutsche Forschungsgemeinschaft as well as the Excellence Initiative of the German federal and state governments for financial help. In addition, we acknowledge the Russian Foundation for Basic Research for the financial support dispatched to A. L. T. through the grant No. 10-03-00155. A. L. T. is deeply thankful to Dr. Michael Gillessen for his kind help with the graphic software.

\section{Appendix A}

Here we discuss how effective magnetic momenta derived from susceptibility measurements can be compared with calculated spin densities. Upon introducing LDA $+U$ theory a comparison was performed against neutron scattering data in the (ordered) low-temperature phase, and reasonable agreement was achieved. ${ }^{37}$ The calculated spin densities correspond to saturation momenta $\mu_{\text {sat }}$ as extracted from sublattice magnetizations measured in the neutron experiments. These momenta are thought to be proportional to the total spins $S$ of the involved magnetic ions. The magnetic susceptibility measurements, if performed in the paramagnetic phase far above the Néel temperature, arrive at effective magnetic momenta $\mu_{\text {eff }} \sim(S(S$ $+1))^{1 / 2}$ so that the saturation momenta are somewhat smaller than the high-temperature effective ones: $\mu_{\text {eff }}=\sqrt{ }[1+(1 / S)] \mu_{\text {sat }}$. Nonetheless, the magnetic susceptibility measurements were performed ${ }^{21}$ in the paramagnetic states just above the respective Néel temperatures. Because of that, extracted values of the effective magnetic momenta $\mu_{\mathrm{eff}}^{*}=797.74\left[\chi_{m} T\right]^{1 / 2}$ carry the temperature dependence of the form: $\sqrt{ }\left[T /\left(T+\left|\theta_{\mathrm{p}}\right|\right)\right]$ due to anti-ferromagnetic correlations in the vicinity of the Néel point. Precisely at the Néel point the following relation holds: $\mu_{\mathrm{eff}} / \mu_{\mathrm{eff}}^{*}$ $=\sqrt{ }\left[1+\left(\left|\theta_{\mathrm{p}}\right| / T_{N}\right)\right]>1$. Unfortunately, the limited range of the temperatures above $T_{\mathrm{N}}$ investigated so far does not allow to extrapolate the $\chi_{\mathrm{m}}{ }^{-1}$ temperature dependence as reliably as needed to get an experimentally substantiated estimate of $\theta_{\mathrm{p}}$. As a workaround, we notice that in the molecular-field approximation both the Néel temperature $T_{\mathrm{N}}$ and the Curie-Weiss temperature $\theta_{\mathrm{p}}$ can be expressed through the effective exchange constants. For the AFM1 state the following holds:

$$
\begin{aligned}
& T_{\mathrm{N}}=3 J_{1}-J_{2} \\
& \theta_{\mathrm{p}}=3 J_{1}+J_{2}
\end{aligned}
$$

so that the conversion coefficient for $\mu_{\text {eff }} / \mu_{\text {effr }}^{*}$ reads $\sqrt{ }\left[2 /\left(1-\left(J_{2} /\right.\right.\right.$ $\left.\left.3 J_{1}\right)\right)$ ]. Here and above we use the signs of the interaction parameters as indicated in Table 1 under the assumption that their ratio is reliable. Uniting the above results the estimates for the saturation magnetic momenta rooting on those for the effective magnetic momenta just above the Néel temperature arrive at the formula:

$$
\mu_{\mathrm{sat}}=\sqrt{\frac{2}{\left(1-\frac{J_{2}}{3 J_{1}}\right)\left(1+\frac{1}{S}\right)}} \mu_{\mathrm{eff}}^{*}
$$

to be used for comparison with the calculated spin densities.

\section{Appendix B}

Here we discuss the possibility of forming different types of insulators with different band composition close to the energy gap through a single mechanism. For the transition-metal monoxides various mechanisms have been proposed to explain their insulating properties, most notably the Coulomb correlation and rhombohedral distortion in the type-II anti-ferromagnetic structure. Originally, introducing the on-site Coulomb correlation term " $U$ " within the manifold of the $3 \mathrm{~d}$ electrons was assumed to yield insulating properties for $\mathrm{MO}(\mathrm{M}=\mathrm{Fe}, \mathrm{Co}$ and $\mathrm{Ni})$, in line with the original formulation of Hubbard theory. Subsequent experimental and theoretical studies evidenced that in these materials the top of their valence bands is spanned predomi- 

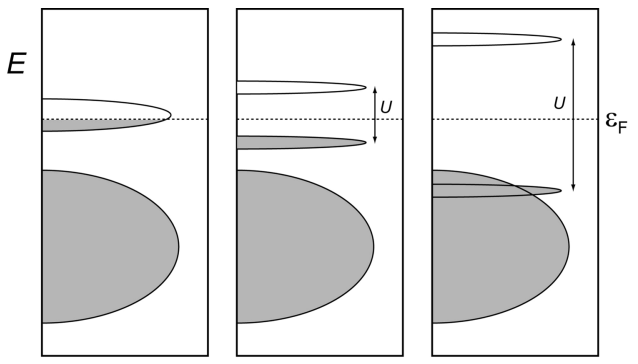

DOS

Figure 5. Density-of-states sketches showing the consequent formation of the metallic (left panel), Mott-Hubbard insulating (middle panel), and charge-transfer insulating (right panel) states of a system under the effect of an increasingly strong Coulomb correlation potential, thereby inducing a splitting of the partially filled ("metallic") band in the left panel into lower (filled) and upper (empty) Hubbard bands in the two other panels.

nantly by the $\mathrm{O} 2 \mathrm{p}$ states $^{7-16}$ as it mutatu mutandis also happens in the Co and Ni carbodiimides ( $2 \mathrm{p}$ states). This is the reason for classifying these materials as charge-transfer insulators (CTI) since they exhibit low-energy excitations of $\mathrm{d}^{n} L \rightarrow \mathrm{d}^{n \pm 1} L^{ \pm}$type. ${ }^{40}$ The general mood is that since the materials exhibiting such an electronic structure are not Mott-Hubbard insulators ${ }^{40}(\mathrm{MHI})-$ their low-energy excitations are not of the $\mathrm{d}^{n} \mathrm{~d}^{n} \rightarrow \mathrm{d}^{n+1} \mathrm{~d}^{n-1}$ typean alternative mechanism of the gap opening (besides the Coulomb correlation of the $3 \mathrm{~d}$ electrons) is to be sought. That tight link between the orbital composition of the bands bordering the gap and the intimate mechanism of the gap opening is not, however, logically nessessary. In other words, the classification of materials ${ }^{40}$ into MHI and CTI according to the character of the low-energy excitations-thereby related to the orbital composition of the allowed energy bands bordering the gap - does not say anything about the way how and why these bands arrived to their final location. The following simple scenario explains how the CTI can appear due to the evolvement of the standard Mott-Hubbard mechanism.

Let us consider a system which in a noncorrelated approximation (e.g., GGA) has the density of states depicted as in Figure 5. Let the lower filled band be a "nonmetallic" band spanned by something like $\mathrm{O} 2 \mathrm{p}$ states and the approximately half-filled band be spanned by metal d states. Applying $U>0$ leads to the Mott-Hubbard splitting of the "metallic" half-filled band into the lower filled and upper empty Hubbard bands. When $U$ is moderate as compared to the width of the original metallic band plus the energy gap between the metallic and nonmetallic filled bands of the original model, the states on top of the filled band of the correlated model are the d states such that the entire picture fits to the definition of MHI: the lowest energy excitations are of the $\mathrm{d}^{n} \mathrm{~d}^{n} \rightarrow \mathrm{d}^{n+1} \mathrm{~d}^{n-1}$ type (Figure 5). If a somewhat stronger Coulomb repulsion is added (larger than the original width of the metallic band plus the energy gap between the metallic and nonmetallic filled bands of the original model), the lower Hubbard band sinks into the wide "nonmetallic" band as shown in Figure 5. In this case the lowest-energy excitations are going to be of the $\mathrm{d}^{n} L \rightarrow \mathrm{d}^{n \pm 1} L^{ \pm}$type, thus suggesting the classification of the material as the CTI one. In this case, however, the CTI behavior of the material is a consequence of the well evolved Mott-Hubbard splitting. No other reason for the gap formation is thus needed in order to obtain the CTI material except the Coulomb correlation potential " $U$ ".

\section{References and Notes}

(1) Roth, W. L. Phys. Rev. 1958, 110, 1333.

(2) Lacour-Gayet, P.; Cyrot, M. J. Phys. C 1974, 7, 400.

(3) Mott, N. F. Proc. Phys. Soc. London, Sec. A 1949) , 62, 416.

(4) Adler, D.; Feinleib, J. Phys. Rev. B 1970, 2, 3112.

(5) Kunz, B.; Surratt, G. T. Solid State Commun. 1978, 25, 2989.

(6) Kunz, B. Int. J. Quant. Chem., Symp. 1981, 15, 487.

(7) Sawatzky, G. A.; Allen, J. W. Phys. Rev. Lett. 1984, 53, 2339.

(8) van Elp, J.; Potze, R. H.; Eskes, H.; Berger, R.; Sawatzky, G. A.

Phys. Rev. B 1991, 44, 1530.

(9) van Elp, J. Ph.D. thesis, University of Groningen: 1991.

(10) Fujimori, A.; Kimizuka, N.; Akahane, T.; Chiba, T.; Kimura, S.; Minami, F.; Siratori, K.; Taniguchi, M.; Ogawa, S.; Suga, S. Phys. Rev. B 1990, 42, 7580.

(11) Bagus, P. S.; Brundle, C. R.; Chuang, T. J.; Wandelt, K. Phys. Rev. Lett. 1977, 39, 1229.

(12) van Elp, J.; Wieland, J. L.; Eskes, H.; Kuiper, P.; Sawatzky, G. A.; de Groot, F. M. F.; Turner, T. S. Phys. Rev. B 1991, 44, 6090.

(13) van Elp, J.; Eskes, H.; Kuiper, P.; Sawatzky, G. A. Phys. Rev. B 1992, 45, 1612 .

(14) Schuler, T. M.; Ederer, D. L.; Itza-Ortiz, S.; Woods, G. T.; Callcott, T. A.; Woicik, J. C. Phys. Rev. B 2005, 71, 115113.

(15) Tran, F.; Blaha, P.; Schwarz, K.; Novák, P. Phys. Rev. B 2006, 74, 155108.

(16) Engel, E.; Schmid, R. N. Phys. Rev. Lett. 2009, 103, 036404.

(17) Mazin, I.; Anisimov, V. I. Phys. Rev. B 1997, 55, 12822.

(18) Adler, D. Rev. Mod. Phys. 1968, 40, 714.

(19) Liu, X. H.; Krott, M.; Müller, P.; Hu, C. H.; Lueken, H.; Dronskowski, R. Inorg. Chem. 2005, 44, 3001.

(20) Krott, M.; Houben, A.; Müller, P.; Schweika, W.; Dronskowski, R. Phys. Rev. B 2009, 80, 024117.

(21) Krott, M.; Liu, X. H.; Fokwa, B. P. T.; Speldrich, M.; Lueken, H.; Dronskowski, R. Inorg. Chem. 2007, 46, 2204.

(22) Liu, X. H.; Stork, L.; Speldrich, M.; Lueken, H.; Dronskowski, R. Chem.-Eur. J. 2009, 15, 1558.

(23) Fei, Y. W.; Ho-kwang Mao, Sci. 1994, 266, 1678.

(24) Noguchi, Y.; Atou, T.; Kondo, T.; Yagi, T.; Syono, Y. Jpn. J. Appl. Phys., part 2 1999, 38, L7.

(25) Hohenberg, P.; Kohn, W. Phys. Rev. 1964, 136, B864.

(26) Kohn, W.; Sham, L. J. Phys. Rev. A 1965, 140, 1133.

(27) Kresse, G.; Hafner, J. Phys. Rev. B 1993, 48, 13115.

(28) Kresse, G.; Furthmüller, J. Phys. Rev. B 1996, 54, 11169.

(29) Kresse, G.; Furthmüller, J. Comput. Mater. Sci. 1996, 6, 15.

(30) Perdew, P.; Burke, S.; Ernzerhof, M. Phys. Rev. Lett. 1996, 77, 3865 .

(31) Blöchl, P. E. Phys. Rev. B 1994, 50, 17953.

(32) Kresse, G.; Joubert, D. Phys. Rev. B 1999, 59, 1758.

(33) Blöchl, P. E.; Jepsen, O.; Andersen, O. K. Phys. Rev. B 1994, 49, 16223.

(34) Anisimov, V. I.; Aryasetiawan, F.; Liechtenstein, A. I. J. Phys.: Condens. Matter 1997, 9, 767.

(35) Dudarev, S. L.; Botton, G. A.; Savrasov, S. Y.; Humphreys, C. J.; Sutton, A. P. Phys. Rev. B 1998, 57, 1505.

(36) Shannon, R. D. Acta Crystallogr. A 1976, 32, 751.

(37) Anisimov, V. I.; Zaanen, J.; Andersen, O. K. Phys. Rev. B 1991, 44, 943.

(38) Hüfner, S.; Osterwalder, J.; Riesterer, T.; Hulliger, F. Solid State Commun. 1984, 52, 793.

(39) A. B. P. Lever. Inorganic Electronic Spectroscopy, 2nd ed; Elsevier: New York, 1984. 418.

(40) Zaanen, J.; Sawatzky, G. A.; Allen, J. Phys. Rev. Lett. 1985, 55,

\section{JP1081033}

